\title{
THE BIRKHOFF_EGERVÁRY-KÖNIG THEOREM FOR MATRICES OVER LATTICE ORDERED ABELIAN GROUPS
}

\author{
By \\ H. SCHNEIDER* (Madison)
}

1. Introduction. A lattice ordered abelian (additive) group (or abelian l-group) is an abelian group $G$ which is also a lattice, and where the infimum and the group operation are related by

$$
\inf \{a+c, b+c\}=\inf \{a, b\}+c,
$$

for $a, b, c \in G$, cf. ClifFord [4], BIRKhoff [1], [3a, Ch. XIV], [3b, Ch. XIII], FuchS $[8, \mathrm{Ch} . \mathrm{V}]$. If the order of $G$ is full (linear), then $G$ is called a fully ordered abelian group (abelian $o$-group) and we write $\min \{a, b\}$ in place of inf $\{a, b\}$. If $G$ is an abelian $l$-group we shall denote by $G^{n n}$ the additive group of $n \times n$ matrices with elements in $G$. A matrix $A \in G^{n n}$ will be called a generalized doubly stochastic matrix (g.d.s. matrix) if

$$
\begin{gathered}
a_{i j} \geqq 0, \quad i, j=1, \ldots, n, \\
\sum_{j=1}^{n} a_{i j}=\sum_{j=1}^{n} a_{j k}, \quad i, k=1, \ldots, n
\end{gathered}
$$

(i. e. all row and column sums are equal). Observe that the 0 matrix in $G^{n n}$ is g.d.s. We denote by $S_{n}$ the symmetric group on $\{1, \ldots, n\}$. If $\sigma \in S_{n}$ and $\varepsilon \in G$, the matrix $P_{\sigma}(\varepsilon)$ is defined by

$$
P_{\sigma}(\varepsilon)_{i j}= \begin{cases}\varepsilon & \text { if } j=\sigma(i), \\ 0, & \text { otherwise. }\end{cases}
$$

If $\varepsilon \geqq 0$, we call $P_{\sigma}(\varepsilon)$ a generalized permutation (g.p.) matrix. We shall prove:

THEOREM. Let $G$ be a lattice ordered abelian group. Every generalized doubly stochastic matrix with elements in $G$ is the sum of generalized permutation matrices.

In the case that $G=\mathbf{Z}$, the integers, this theorem is due to KöNIG, $1916[9$, Theorem F], see also KőNIG [10, p. 239, Theorem B], MiRsKY [15, p. 186, Theorem 11.1.5]. For $G=\mathbf{R}$, the real numbers, the theorem is due to BIRKHOFF 1946 [2], see also MARCUs-MINC [13, p. 97, Theorem 1.7], MIRSKY [15, p. 192, Theorem 11.3.1] and MirSKY [14], where many other references to Birkhoff's theorem and its proofs may be found. It should also be noted that in 1931 EGERVÁRY [5, Theorem II] proved a result for integral matrices which is more general than Konnig's theorem. He observed that by continuity considerations his theorem may be shown to hold for real matrices. Thus in this way one obtains a result which contains Birkhoff's theorem.

* This research has been partly supported by NSF Grant MPS 73-08618 AO2. 
To obtain a result which contains both Birkhoff's theorem and Kơnig's theorem it is enough to prove our Theorem in the case that $G$ is an abelian $o$-group. Though a proof of this case is essentially the same as the proof of Birkhoff's theorem given in MARCus-Minc [13, pp. 97, 98], we have not found the result formulated for abelian $o$-groups, or in such a manner to contain both the case $G=\mathbf{R}$ and $G=\mathbf{Z}$. (BIRKhoff [3a, p. 266, Ex. 4] remarks that the theorem is valid for matrices with elements in a fully ordered field, which is close.)

To extend the theorem to abelian l-groups we use an embedding result due to ClIFFORD [4, Theorem 2] who remarks that this theorem is a combination of Theorems 4 and 11 of LoRENZEN [11], which, however, do not deal with $l$-groups explicitly. The Clifford-Lorenzen theorem states: Let $G$ be an abelian $l$-group. Then there exists a family $G(\tau), \tau \in T$, of abelian $o$-groups, and an isomorphism $g \rightarrow(g(\tau))_{\tau \in T}$ of $G$ into the direct product $(G(\tau))_{\tau \in T}$ such that for $a, b \in G$ and $c=\inf \{a, b\}$ we have $c(\tau)=\min \{a(\tau), b(\tau)\}$. For this theorem, see BrRKHOFF [3b, p. 309, Theorem 22] and for a generalization, LORENZEN [12, Theorem 13]. Following SIK [18], and RIBENBOIM [16] we call a family of $G(\tau), \tau \in T$, together with an isomorphism with the above property, a realization of $G$. (An example of an abelian $l$-group which is not the direct sum or direct product of $o$-groups is given in S̆IK [17].) With the above remarks the proof of our theorem is very straightforward.

2. Proofs. If $\sigma$ is a permutation in $S_{n}$, and $A \in G^{n n}$ then we define the diagonal $D_{\sigma}$ to be the set $\left\{a_{1 \sigma(1)}, \ldots, a_{n \sigma(n)}\right\}$. Part (a) of the proof of the Lemma below requires the Frobenius-Kónig Theorem, see Frobenius [7], König [10, p. 240, Theorem E], Marcus-Mrnc [13, p. 97, 1.7.1], Mirsky [15, p. 189, Cor. 11.2.6]: If every diagonal of $A \in G^{n n}$ has a zero element, then $A$ contains a zero submatrix of order $p \times(n+1-p)$, for some $p, 1 \leqq p<n$.

LEMMA. Let $G$ be a lattice ordered abelian group and let $A \in G^{n n}$ be a generalized doubly stochastic matrix. If inf $D_{\sigma}=0$, for every, $\sigma \in S_{n}$, then $A=0$.

Proof. (a) First assume that $G$ is an abelian $o$-group. Since $\min D_{\sigma}=0$, for every $\sigma \in S_{n}$, every diagonal of $A$ has a 0 member. Hence, by Frobenius-König, $A$ has a 0 submatrix of order $r \times(n+1-r)$, for some $r, 1 \leqq r<n$. Without loss of generality, we may assume that $a_{i j}=0, i=1, \ldots, r, j=r, \ldots, n$. Let

Then

$$
\sum_{j=1}^{n} a_{i j}=s=\sum_{j=1}^{n} a_{i j}, \quad i=1, \ldots, n \text {. }
$$

$$
r s=\sum_{i=1}^{r} \sum_{j=1}^{n} a_{i j}=\sum_{i=1}^{r} \sum_{j=1}^{r-1} a_{i j} \leqq \sum_{j=1}^{r-1} \sum_{i=1}^{n} a_{i j}=(r-1) s,
$$

whence $s=0$. But then $a_{i j}=0, i, j=1, \ldots, n$, and so $A=0$.

(b) Now suppose that $G$ is an arbitrary abelian l-group. By the Clifford-Lorenzen theorem there exists a realization $G \rightarrow(G(\tau))_{\tau \in T}$, where the $G(\tau)$ are abelian o-groups. We put $A(\tau)=\left(a_{i j}(\tau)\right) \in G(\tau)^{n n}$, and for $\sigma \in S_{n}$ we denote by $D_{\sigma}(\tau)$ the diagonal $\left\{a_{1 \sigma(1)}(\tau), \ldots, a_{n \sigma(n)}(\tau)\right\}$ of $A(\tau)$. Then, for each $\tau \in T$,

$$
\min D_{\sigma}(\tau)=\left(\inf D_{\sigma}\right)(\tau)=0,
$$


whence by Part (a) of this proof $A(\tau)=0$, since $A(\tau)$ is a g.d.s. matrix in $G(\tau)^{n !}$. It follows that $A=0$.

PROOF OF THE THEOREM. We use induction on the number $k$ of $\sigma \in S_{n}$ with inf $D_{\sigma}>0$. If $k=0$, then by the Lemma, $A=0$ and the result holds. So suppose that $k>0$, and the result holds for matrices $A^{\prime}$ with fewer than $k$ diagonals with non-zero infimum. Let $\sigma \in S_{n}$ be such that $d=\inf D_{\sigma}>0$, and put $A^{\prime}=A-P_{\sigma}(d)$. Then $A^{\prime}$ is g.d.s., and for every $\pi \in S_{n}$, inf $D_{\pi}^{\prime} \leqq \inf D_{\pi}$. Also inf $D_{\sigma}^{\prime}=\inf D_{\sigma}-d=0$, where $D_{\pi}^{\prime}, \pi \in S_{n}$, denotes the diagonal of $A^{\prime}$ corresponding to $\pi$. Hence $A^{\prime}$ has fewer than $k$ diagonal's with positive infimum, and by inductive assumption, $A^{\prime}$ is the sum of g.p. matrices. It follows that $A=A^{\prime}+P_{\sigma}(d)$ is also a sum of g.p. matrices. The theorem is proved.

3. If $G=\mathbf{R}$, then it is well known that every g.d.s. matrix can be expressed as the sum of at most $\left(n^{2}-2 n+2\right)$ g.p. matrices $P_{\sigma}\left(\varepsilon_{\sigma}\right)$, with $\varepsilon_{\sigma}>0$, FARAHAT-MrRSKY [6], cf. MrRsky [14], Marcus-Minc [13, pp. 94-100], and (incidentally) this bound is best possible. We give an example of an abelian $l$-group $G$ and a g.d.s. matrix $A \in G^{n n}$, which has a unique representation $A=\sum_{\sigma \in S_{n}} P_{\sigma}\left(\varepsilon_{\sigma}\right)$ and $\varepsilon_{\sigma}>0$, for all $n !$ permutations $\sigma$. We put $G=Z^{m}$, where $m=n$ !, the direct sum of $n$ ! copies of the integers $\mathbf{Z}$, and we index the copies of $\mathbf{Z}$ by $S_{n}$. Thus the elements of $G$ are the $n$ ! tuples $(a(\tau))_{\tau} \in S_{n}$.

We next define $A \in G^{n n}$ by

$$
a_{i j}(\tau)= \begin{cases}1, & \text { if } j=\tau(i), \\ 0, & \text { otherwise. }\end{cases}
$$

Then $A$ is a g.d.s. matrix for clearly $a_{i j} \geqq 0, i, j=1, \ldots, n$ and, for each $\tau \in S_{n}$

$$
\sum_{j=1}^{n} a_{i j}(\tau)=\sum_{j=1}^{n} a_{j i}(\tau)=1
$$

If, $\sigma, \tau \in S_{n}$, let $A=\sum_{\sigma \in S_{n}} P_{\sigma}\left(\varepsilon_{\sigma}\right)$. Then

$$
\varepsilon_{\sigma}(\tau) \leqq \inf D_{\sigma}(\tau)=\inf \left\{a_{1 \sigma(1)}(\tau), \ldots, a_{n \sigma(n)}(\tau)\right\}=0, \text { if } \quad \sigma \neq \tau
$$

and it follows that

$$
\varepsilon_{\sigma}(\sigma)=1, \quad \varepsilon_{\sigma}(\tau)=0, \quad \text { if } \quad \sigma \neq \tau
$$

We now write out our example in full, for the case that $n=3$ and the permutations in $S_{n}$ are arranged in order

$$
(1,2,3),(1,3,2),(2,1,3),(2,3,1),(3,1,2),(3,2,1),
$$

where $(i, j, k)$ is the permutation $\sigma$ for which $\sigma(1)=i, \sigma(2)=j, \sigma(3)=k$. Then $A$ is

$$
\left(\begin{array}{lll}
(1,1,0,0,0,0) & (0,0,1,1,0,0) & (0,0,0,0,1,1) \\
(0,0,1,0,1,0) & (1,0,0,0,0,1) & (0,1,0,1,0,0) \\
(0,0,0,1,0,1) & (0,1,0,0,1,0) & (1,0,1,0,0,0)
\end{array}\right)
$$




\section{References}

[1] G. Birkhoff, Lattice-ordered groups, Ann. of. Math., (2) 43 (1942), 298-331.

[2] G. BrRkhoff, Tres observaciones sobre el algebra lineal, Univ. Nac. Tucuman Rev., Sev. A, 5 (1946), 147-150.

[3] G. Birkhoff, Lattice theory, Amer. Math. Soc. Colloq. Pub]. Vol. 25, Providence, R. I., (a) 2nd Edn. (1948), (b) 3rd Edn. (1967).

[4] A: H. Clifford, Partially ordered abelian groups, Annals Math., (2) 41 (1940), 465-473.

[5] E. EgerváRY (a) Mátrixok kombinatórikus tulajdonságairól, Mat. Fiz. Lapok, 38 (1931), $16-28$ (in Hungarian).

(b) On combinatorial properties of matrices, George Washington Univ. Logistic Papers, 11 (1955) (transl. H. W. Kuhn).

[6] H. K. Farakat and L. Mirsky, Permutation endomorphisms and refinement of a theorem of Birkhoff, Proc. Cambridge Phil. Soc., 56 (1960), 322-328.

[7] G. F. Frobenius, Über zerlegbare Determinanten.

(a) Sitzungsber. Preuss. Akad. Wiss. (Berlin, 1917), $274-277$.

(b) Ges. Abh. \# 102, Vol. 3, 701-704, Springer-Verlag (Berlin, 1968).

[8] L. Fuchs, Partially ordered algebraic systems, Addison-Wesley Pub. Co. (Reading, Mass., 1963).

[9] D. KóNIG, (a) Über Graphen und ihre Anwendung auf Determinantentheorie und Mengenlehre, Math. Ann., 77 (1916), 453-465.

(b) Gráfok és alkalmazásuk a determinánsok és halmazok elméletében, Math. és Termész. Ért., 34 (1916), 104-119 (in Hungarian).

[10] D. KóNIG, Theorie der endlichen und unendlichen Graphen, (a) Akad. Verlagsges. (Leipzig, 1936), (b) Chelsea Pub. Co. (New York, 1950).

[11] P. Lorenzen, Abstrakte Begründung der multiplikativen Idealtheorie, Math. Z., 45 (1939), $533-553$.

[12] P. Lorenzen, UUber halbgeordnete Gruppen, Math. Z., 52 (1949), 483-526.

[13] M. MARcus-H. Minc, A survey of matrix theory and matrix inequalities, Allyn \& Bacon (Boston, 1964).

[14] L. Mirsky, Results and problems in the theory of doubly-stochastic matrices, $Z$. Wahrscheinlichkeits., 1 (1963), 319-334.

[15] L. MrRsky, Transversal Theory, Math. in Science and Eng. Vol. 75, Academic Press (1971).

[16] P. Rubendoim, Un théorème de réalisation de groupes réticulès, Pacific J. Math., 10 (1960), $305-308$.

[17] F. ŠIK, Über Summen einfach geordneter Gruppen, Czechoslovak Math. J., 8 (1953), $22-53$.

[18] F. SIK, Über subdirekte Summen geordneter Gruppen, Czechoslovak Math. J., 10 (1960), $400-424$.

(Received April 27, 1976)

UNIVERSITY OF WISCONSIN

MATHEMATICS DEPARTMENT

MADISON, WISCONSIN 53706

USA 